\title{
Enzymatic activity of a glycosyltransferase KanM2 encoded in the kanamycin biosynthetic gene cluster
}

\author{
Fumitaka Kudo $^{1}$, Hilda Sucipto ${ }^{2}$ and Tadashi Eguchi ${ }^{2}$
}

The Journal of Antibiotics (2009) 62, 707-710; doi:10.1038/ja.2009.107; published online 13 November 2009

Keywords: aminoglycoside antibiotics; biosynthesis; glycosyltransferase; kanamycin; paromamine

Kanamycin, discovered by Umezawa in 1957, is the most well-known 2-deoxystreptamine (2DOS)-containing aminoglycoside antibiotic. ${ }^{1}$ Three structurally related kanamycins were identified from the producer Streptomyces kanamyceticus (Figure 1). Extensive biosynthetic studies of this class of aminoglycoside antibiotics, especially butirosin and neomycin, clarified that a unique aminocyclitol, 2DOS, is biosynthesized from D-glucose 6-phosphate by three crucial enzymes, 2-deoxy-scyllo-inosose (2DOI) synthase, L-glutamine (Gln):2DOI aminotransferase and 2-deoxy-scyllo-inosamine dehydrogenase. $^{2,3}$ After the 2DOS formation, uridine $5^{\prime}$-diphospho- $N$-acetylglucosamine (UDP-GlcNAc):2DOS $N$-acetylglucosaminyltransferase catalyzes the glycosylation of 2DOS to give $\mathrm{N}$-acetylparomamine, which is then deacetylated by $N$-acetylparomamine deacetylase to yield paromamine. ${ }^{4}$ Paromamine is believed to be a branching biosynthetic intermediate, which is converted to either 4,6-disubstituted 2DOS aminoglycosides, such as kanamycin and gentamicin, or 4,5-disubstituted 2DOS aminoglycosides, such as neomycin and butirosin. Therefore, the regio-specificities and substrate specificities of glycosyltransferases that recognize paromamine as a glycosyl acceptor determine the core structures of aminoglycoside antibiotics.

The biosynthetic gene cluster for kanamycin, comprising 24 open reading frames, was identified in $2004 .^{5}$ Two other research groups also deposited independently identical kanamycin biosynthetic genes by different symbols in the public DNA databases simultaneously. ${ }^{6,7}$ To prevent confusion, herein we use the btr gene-based names, which were used systematically by the Piepersberg group and also in our recent review. ${ }^{8,9}$ The entire kanamycin biosynthetic gene cluster was expressed in Streptomyces venezuelae and the resultant recombinant strain was reported to produce kanamycin $\mathrm{A}$, which indicates that the kan genes are responsible for the biosynthesis of kanamycins. ${ }^{10}$ The kanC, kanS1, kanE, kanM1 and kanN genes expressing Streptomyces lividans reportedly produce paromamine, confirming that these genes, respectively, encode 2DOI synthase, Gln:2DOI aminotransferase, 2-deoxy-scyllo-inosamine dehydrogenase, UDP-GlcNAc:2DOS N-acetylglucosaminyltransferase and $\mathrm{N}$-acetylparomamine deacetylase (Figure 1). ${ }^{11}$ Among these, the kanC gene was expressed in Escherichia coli and the recombinant KanC protein was confirmed to be 2DOI synthase. $^{6}$ Recombinant KanM1 protein was also reported to have weak glycosyltransferase activity with 2DOS as a glycosyl acceptor, and TDP-glucose or GDP-mannose as a glycosyl donor. ${ }^{12}$ However, this KanM1 activity is contradictory to the presumed enzymatic function as suggested by the above-mentioned heterologous expression of UDP-GlcNAc:2DOS $\mathrm{N}$-acetylglucosaminyltransferase in S. lividans. Detailed enzymatic analysis is thus necessary to elucidate the substrate specificity of KanM1 on using a pure enzyme.

In this study, we heterologously expressed another glycosyltransferase gene, kanM2, in the kan gene cluster and investigated its enzymatic activities, which were expected to be catalysis of the glycosylation of a pseudo-disaccharide, paromamine, to yield a pseudo-trisaccharide. To obtain soluble KanM2 protein expressed in E. coli, a low expression temperature $\left(15^{\circ} \mathrm{C}\right)$ was critically important after addition of isopropyl $\beta$-D-thiogalactoside (final concentration $0.5 \mathrm{~mm}$ ). The cell-free extract of the KanM2-expressing E. coli was treated with $2 \mathrm{~mm}$ of paromamine as a glycosyl acceptor, and $2 \mathrm{~mm}$ of UDP-Glc or UDPGlcNAc as a glycosyl donor. After incubation at $28^{\circ} \mathrm{C}$ for $18 \mathrm{~h}$, the enzymatic reactions were quenched by addition of ethanol. The resulting mixture was treated with 2,4-dinitrofluorobenzene to convert aminoglycosides into $\mathrm{N}$-dinitrophenyl derivatives, which show UV-Vis absorption at $350 \mathrm{~nm}$. Consequently, only a reaction mixture of paromamine and UDP-Glc gave a new peak with $40 \%$ conversion from the substrate paromamine in HPLC traces (Figures 2a and b). Liquid chromatography-electrospray ionizationMS analysis (negative mode) of the sample showed the new peak with $\mathrm{m} / \mathrm{z}$ 982.4, which corresponded to $[\mathrm{M}-\mathrm{H}]^{-}$for the derivative of glucosylated paromamine (Figure 2c).

Next, we attempted to isolate the KanM2 reaction product from paromamine and UDP-Glc, and determine the chemical structure.

1Department of Chemistry, Tokyo Institute of Technology, Tokyo, Japan and ${ }^{2}$ Department of Chemistry and Materials Science, Tokyo Institute of Technology, Tokyo, Japan Correspondence: Professor T Eguchi, Department of Chemistry and Materials Science, Tokyo Institute of Technology, 2-12-2 0-okayama, Meguro-ku, Tokyo 151-8551, Japan. E-mail: eguchi@cms.titech.ac.jp

Received 17 August 2009; revised and accepted 20 October; published online 13 November 2009 

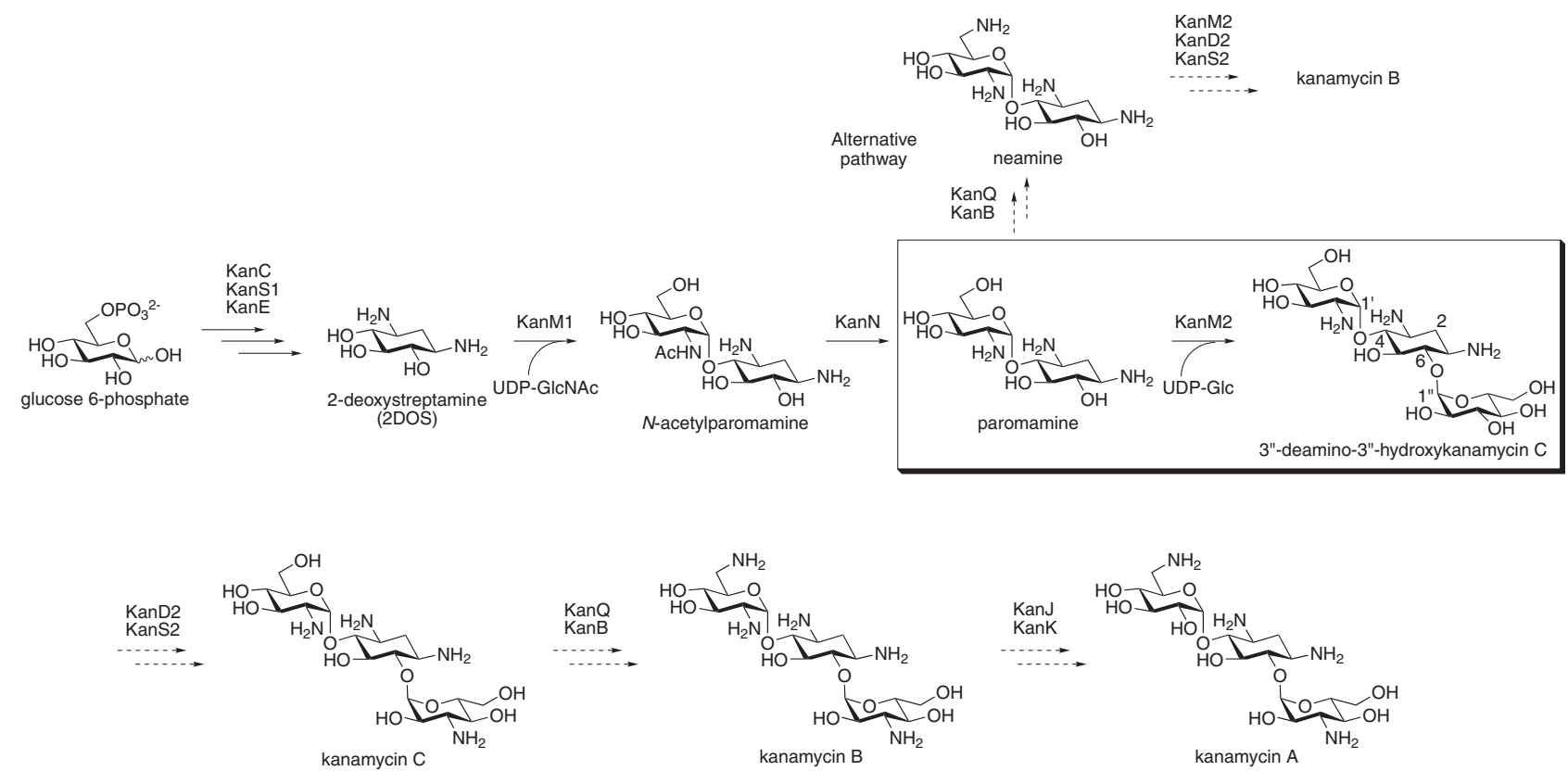

Figure 1 Proposed biosynthetic pathway for kanamycin.

Ammonium sulfate fractionation between 10 and 40\% saturation was effective in recovering most of the enzymatic activity from the cell-free extract of KanM2-expressing E. coli, although its activity was significantly decreased after a standard dialysis probably because of the instability of the enzyme. We then used the $10-40 \%\left(\mathrm{NH}_{4}\right)_{2} \mathrm{SO}_{4}$ fractionated solution just after the fractionation, paromamine ( $3 \mathrm{~mm}, 10 \mathrm{mg}$ ), and UDP-Glc $(4.7 \mathrm{~mm}, 29 \mathrm{mg}$ ) in $50 \mathrm{~mm}$ HEPES buffer (pH 8.0) containing $1 \mathrm{~mm} \mathrm{MgCl}_{2}$ and $10 \%$ glycerol (total $10 \mathrm{ml}$ ) at $28{ }^{\circ} \mathrm{C}$ for $24 \mathrm{~h}$ for a large-scale enzymatic reaction. To ensure maximum consumption of the starting material, the reaction mixture was further treated under identical enzymatic conditions using freshly prepared enzyme solution $(0.25 \mu \mathrm{l})$ and UDP-Glc $(5-10 \mathrm{mg})$ nine times. Consequently, $84 \%$ of paromamine was converted to the new glycosylated product, which was obtained as the sulfate form of aminoglycoside $(7.9 \mathrm{mg})$ by ion exchange chromatography. HR-FABMS (positive mode, glycerol) of the compound gave an $\mathrm{m} / \mathrm{z}$ of 486.2307, which corresponds to the calculated mass for $\mathrm{C}_{18} \mathrm{H}_{36} \mathrm{O}_{12} \mathrm{~N}_{3}$ (486.2299), $[\mathrm{M}+\mathrm{H}]^{+}$of glucosylated paromamine. ${ }^{1}$ $\mathrm{H}-\mathrm{NMR}$ analysis of the product showed two anomeric protons and a methylene of the 2DOS moiety, although the other signals were very complex for assignment (see Supplementary information). Spectra of the compound obtained by total correlation spectroscopy (TOCSY) indicated that the signals for $\mathrm{H}-1$ and $\mathrm{H}-3$ of $2 \mathrm{DOS}$ at 3.3 p.p.m., those for $\mathrm{H}-6$ at 3.6 p.p.m., and those for $\mathrm{H}-4$ and $\mathrm{H}-5$ at 3.7 p.p.m. are correlated with the signals for $\mathrm{H}-2 \mathrm{ax}$ and $\mathrm{H}-2 \mathrm{eq}$ at 1.7 and 2.3 p.p.m., respectively (data not shown). The use of ${ }^{1} \mathrm{H}-{ }^{1} \mathrm{H}-\mathrm{COSY}$, heteronuclear multiple quantum coherence and heteronuclear multiple bond coherence facilitated assignment of ${ }^{13} \mathrm{C}$ chemical shifts for $\mathrm{C1}^{\prime \prime}, \mathrm{Cl}^{\prime}, \mathrm{C}-2^{\prime}$, C-1 or C-3, C-4, C-5 and C- 6 . Consequently, a glycosidic linkage between $\mathrm{Cl}^{\prime \prime}$ and $\mathrm{C}-6$ was confirmed in addition to the $\mathrm{C1}^{\prime}-\mathrm{O}-\mathrm{C} 4$ bond of the paromamine moiety. Therefore, the KanM2 reaction product from paromamine and UDP-Glc appeared to be $3^{\prime \prime}$-deamino$3^{\prime \prime}$-hydroxykanamycin C.

Presumably, $3^{\prime \prime}$-deamino- $3^{\prime \prime}$-hydroxykanamycin $\mathrm{C}$ is a biosynthetic intermediate leading to kanamycin B and kanamycin A (Figure 1).
A disruptant mutant of the kanQ gene homolog encoding a FAD-dependent dehydrogenase in the tobramycin and apramycin producer Streptomyces tenebrarius accumulated 3'-deoxy-carbamoylkanamycin C. ${ }^{13}$ This accumulation suggests that the KanM2 homologous protein, TobM2, in tobramycin biosynthesis recognizes paromamine as a substrate to produce $3^{\prime \prime}$-deamino- $3^{\prime \prime}$-hydroxykanamycin $\mathrm{C}$, which would be dehydrogenated and transaminated to give kanamycin C. An $\mathrm{NAD}^{+} / \mathrm{NADP}^{+}$-dependent dehydrogenase, KanD2, and an aminotransferase, KanS2, are presumably responsible for this transformation based on bioinformatic analysis of the gene clusters for 2DOS-containing aminoglycoside antibiotics. ${ }^{9}$ Alternatively, this set of enzymes might be responsible for the formation of NDP-3-amino-3-deoxyglucose (kanosamine), which is another possible glycosyl donor in the KanM2 reaction, to afford kanamycin C. ${ }^{11}$ KanS2 is a protein that is homologous to KanS1 ( $37 \%$ identity $/ 51 \%$ positive), which is involved in the double transamination in 2DOS biosynthesis. The difference in substrate specificity of these aminotransferases is an interesting issue to be resolved.

A FAD-dependent dehydrogenase, KanQ, and an aminotransferase, $\mathrm{KanB}$, remaining in the kan gene cluster are apparently responsible for the transamination at C- 6 . The substrate specificity of this set of enzymes is also interesting because the homologous enzymes were reported to recognize mainly the glucosamine moiety and are responsible for the transaminations at C-6 in neomycin biosynthesis. ${ }^{14,15}$ Thus, these enzymes might convert paromamine to neamine, which could be glycosylated by KanM2 to yield kanamycin B. Thus, the substrate specificity of KanM2 is a key determinant for the production of kanamycin analogs in the producer strain.

Finally, kanamycin B is converted to the end product kanamycin A. A feeding experiment of ${ }^{D}-\left[{ }^{14} \mathrm{C}\right]$ glucosamine into the kanamycin producer revealed that the labeled glucosamine was incorporated only into the 6-amino-6-deoxy-glucose moiety of kanamycin A, ${ }^{16,17}$ which suggests that the deamination reaction at C-2 of the glucosamine moiety in kanamycin $\mathrm{B}$ is involved in the biosynthesis of 


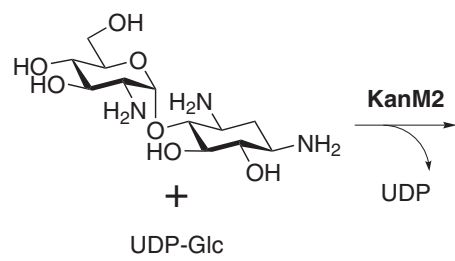

UDP-GIC

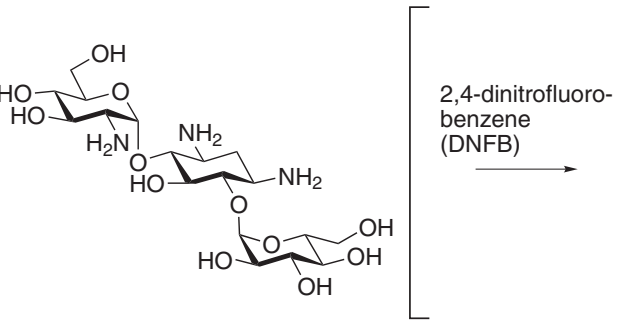

For detection at $350 \mathrm{~nm}$ $[\mathrm{M}-\mathrm{H}]^{-} 982$
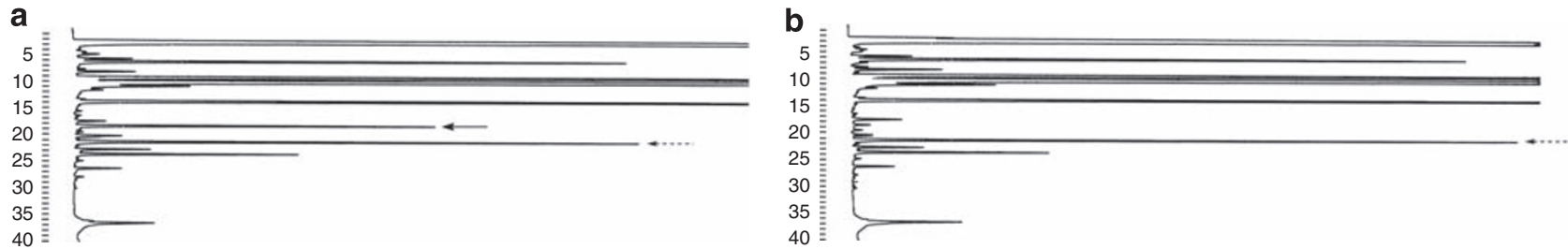

C
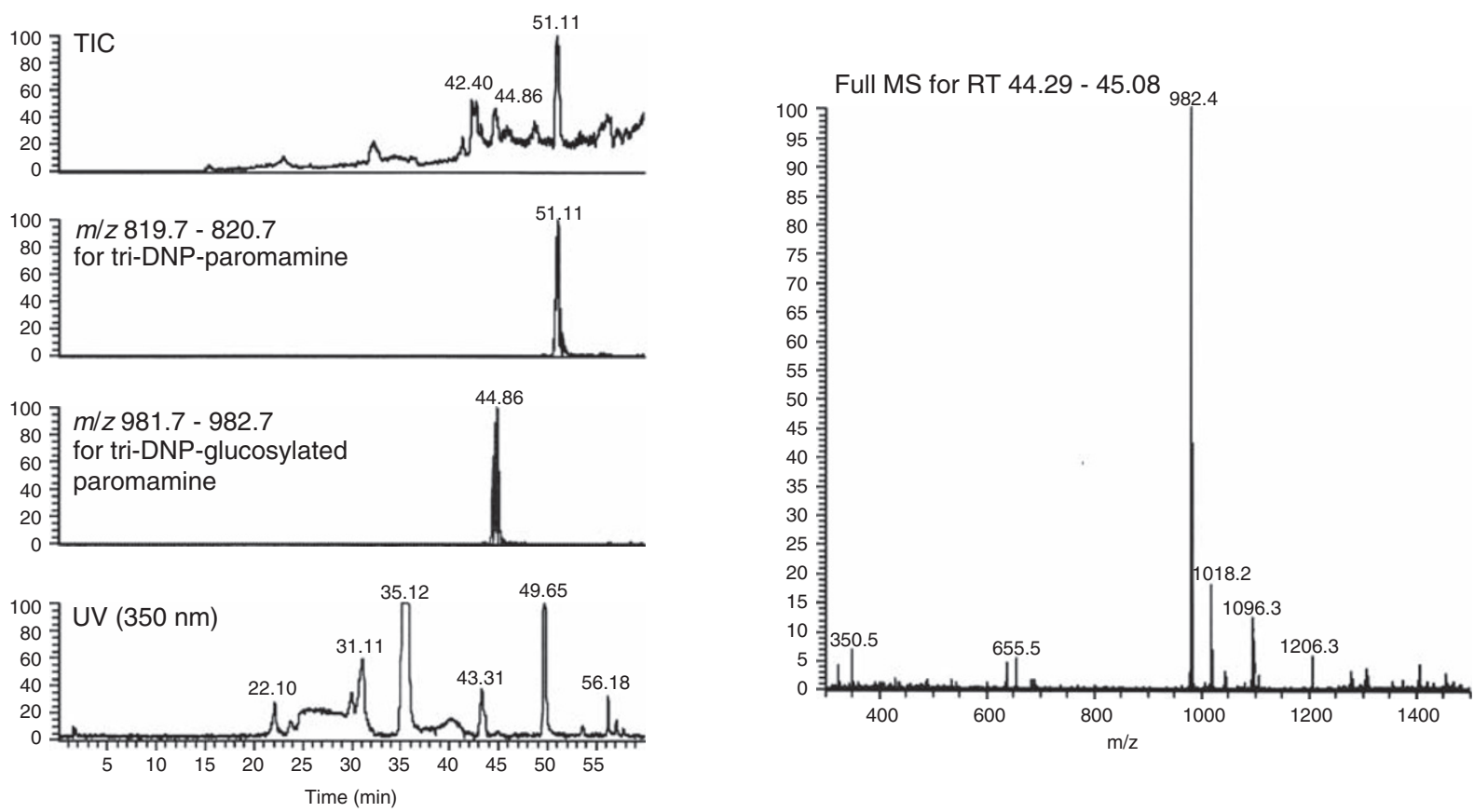

Figure 2 KanM2 reaction of paromamine with UDP-Glc. After the enzymatic reactions, aminoglycoside compounds were converted to the dinitrophenyl derivatives $(350 \mathrm{~nm})$ for detection by HPLC (refer the Supplementary information for details). (a) KanM2's reaction with paromamine and UDP-Glc; (b) KanM2's reaction with paromamine (without addition of UDP-GIc); (c) liquid chromatography-electrospray ionization-MS (LC-ESI-MS) analysis (negative mode) of the same solution in (a). The solid arrow indicates the KanM2 reaction product and the broken arrow denotes the paromamine that remained in the enzymatic solution. Several unidentified peaks were observed in the HPLC traces, which might have been derived from impurities in the cell-free extract (CFE) of the KanM2-expressing E. coli.

kanamycin A. Two unassigned hypothetical proteins, KanJ (a putative phytanoyl-CoA dioxygenase family protein) and KanK (a putative NAD-dependent protein), are apparently responsible for this unprecedented deamination reaction, which is presumably accomplished through either transamination/dehydrogenation or oxidation (and subsequent hydrolysis of an imine intermediate)/dehydrogenation chemistry.

In conclusion, we clarified that KanM2 catalyzes the glucosylation of paromamine with UDP-Glc to yield $3^{\prime \prime}$-deamino- $3^{\prime \prime}$-hydroxykanamycin C. This result strongly supports the proposed stepwise biosynthetic pathway via paromamine as an intermediate (Figure 1).
However, a detailed biochemical analysis of KanM2 including substrate specificity remains to be done. The amino acid sequences of KanM2 and KanM1 show 33\% identity and 54\% positivity with each other. It is therefore interesting to investigate how these glycosyltransferases distinguish glycosyl acceptors 2DOS or paromamine, and glycosyl donors UDP-GlcNAc or UDP-Glc. In addition, understanding such biochemical properties of glycosyltransferases will provide important information to engineer proteins for glycodiversification in order to create diverse structural glycosides using enzymes. ${ }^{18}$ Purification of KanM1 and KanM2 for detailed biochemical studies is currently in progress. 


\section{ACKNOWLEDGEMENTS}

This work was supported in part by KAKENHI 21710224 (FK).

1 Umezawa, H. et al. Production and isolation of a new antibiotic: kanamycin. J. Antibiot. 10, 181-188 (1957)

2 Kudo, F., Yamamoto, Y., Yokoyama, K., Eguchi, T. \& Kakinuma, K. Biosynthesis of 2-deoxystreptamine by three crucial enzymes in Streptomyces fradiae NBRC 12773. J. Antibiot. 58, 766-774 (2005).

3 Kudo, F. \& Eguchi, T. Biosynthetic enzymes for the aminoglycosides butirosin and neomycin. Methods Enzymol. 459, 493-519 (2009).

4 Yokoyama, K., Yamamoto, Y., Kudo, F. \& Eguchi, T. Involvement of two distinct $\mathrm{N}$-acetylglucosaminyltransferases and a dual-function deacetylase in neomycin biosynthesis. Chembiochem 9, 865-869 (2008).

5 Yanai, K. \& Murakami, T. The kanamycin biosynthetic gene cluster from Streptomyces kanamyceticus. J. Antibiot. 57, 351-354 (2004).

6 Kharel, M. K. et al. A gene cluster for biosynthesis of kanamycin from Streptomyces kanamyceticus: comparison with gentamicin biosynthetic gene cluster. Arch. Biochem. Biophys. 429, 204-214 (2004).

7 Piepersberg, W. Cloning and sequencing of the kanamycin biosynthetic gene cluster from Streptomyces kanamyceticus DSM 40500 Accession No. AJ628422 (2006).

8 Piepersberg, W., Aboshanab, K. M., Schmidt-Beissner, H. \& Wehmeier, U. F. in Aminoglycoside Antibiotics. The Biochemistry and Genetics of Aminoglycoside Producers (ed Arya, D. P.) 15-118 (John Wiley \& Sons, Inc., Hoboken, NJ, 2007).
9 Kudo, F. \& Eguchi, T. Biosynthetic genes for aminoglycoside antibiotics. J. Antibiot. 62, 471-481 (2009).

10 Thapa, L. P. et al. Heterologous expression of the kanamycin biosynthetic gene cluster (pSKC2) in Streptomyces venezuelae YJ003. Appl. Microbiol. Biotechnol 76, 1357-1364 (2007).

11 Nepal, K. K., Oh, T. J. \& Sohng, J. K. Heterologous production of paromamine in Streptomyces lividans TK24 using kanamycin biosynthetic genes from Streptomyces kanamyceticus ATCC12853. Mol. Cells 27, 601-608 (2009).

12 Park, S. H. et al. Expanding substrate specificity of GT-B fold glycosyltransferase via domain swapping and high-throughput screening. Biotechnol. Bioeng. 102, 988-994 (2009).

13 Yu, Y., Hou, X., Ni, X. \& Xia, H. Biosynthesis of 3'-deoxy-carbamoylkanamycin C in a Streptomyces tenebrarius mutant strain by tacB gene disruption. J. Antibiot. 61, 63-69 (2008).

14 Huang, F. et al. Elaboration of neosamine rings in the biosynthesis of neomycin and butirosin. Chembiochem 8, 283-288 (2007).

15 Kudo, F., Kawashima, T., Yokoyama, K. \& Eguchi, T. Enzymatic preparation of neomycin C from ribostamycin. J. Antibiot. 62, 643-646 (2009).

16 Kojima, M., Yamada, Y. \& Umezawa, H. Biosynthesis of kanamycins. I. Incorporation of glucose-14C or glucosamine glucosam $14 \mathrm{C}$ into kanamycins and kanamycin-related compounds. Agric. Biol. Chem 32, 467-473 (1968).

17 Rinehart, K. L. Jr \& Stroshane, R. M. Biosynthesis of aminocyclitol antibiotics. J. Antibiot. 29, 319-353 (1976).

18 Thibodeaux, C. J., Melançon, C. E. III \& Liu, H. W. Natural-product sugar biosynthesis and enzymatic glycodiversification. Angew Chem. Int. Ed. 47, 9814-9859 (2008).

Supplementary Information accompanies the paper on The Journal of Antibiotics website (http://www.nature.com/ja) 